Review

\title{
SPECIFICATIONS OF THE COVID-19 INFECTION IN CHILDREN
}

\author{
A.Vasileva $^{1 *}$, Iv. Chakarov ${ }^{2}$, P. Chakarova ${ }^{1}$ \\ ${ }^{1}$ Clinic of Pediatrics, UMHAT "Prof. doctor St. Kirkovich", Stara Zagora, Bulgaria \\ ${ }^{2}$ Clinic of Childhood Clinical Hematology and Oncology UMHAT "Tsaritsa Yoanna-ISUL", Sofia, \\ Bulgaria
}

\begin{abstract}
First encountered in the 2019 COVID-19 virus has a high transmission capacity, and children are not spared. Therefore, we set a goal, referring to the experience of the countries in which the infection spread the earliest and widest, to make a summary of the characteristics of COVID-19 in the pediatric population.

Of course, the most significant thing is the variation in the clinical manifestation, the correct assessment of the clinical risk of lung damage, as well as the laboratory and imaging methods of examination that support a reliable diagnosis.

The therapy section of the article describes all the possible strategies for therapeutic behavior.

Despite the rapidly growing theoretical information and shared clinical experience, the article could be a foundation on which to add and expand theoretical and practical knowledge.
\end{abstract}

Key words: COVID-19, SARS, ACE-2 receptors.

\section{INTRODUCTION}

The incidence of COVID-19 in children has always been and will always be very different from that in adults. In December 2019 the coronavirus was isolated in Wuhan, China. It has a very high transmission capacity and is known as SARS-COVID-19. The need for good knowledge reflects on public health. Although the number of infected children worldwide, and in particular nationally, is currently small, children are not protected from infection.

\section{STRUCTURE OF THE CORONAVIRUS}

Coronaviruses are a large family of viruses and a subset of Coronaviridae, belonging to the order Nidovirales, the kingdom of RIboviria, which range from the common cold virus to more severe pathogens such as SARS, MERS, and COVID-19.

Encountered in 2019, the COVID-19 has a high transmission capacity and it is already clear that it is SARS-COV-2. [1,2] All

Correspondence to: $D r$. A.Vasileva, Assistant at Clinic of Pediatrics, UMHAT "Prof. Dr. St. Kirkovich”, Stara Zagora, Bulgaria, email: ani_asken@abv.bg
Nidovirales viruses are RNA viruses with very large genomes, and Coronaviridae has the largest known RNA genome and contains approximately 30 kilobases $(\mathrm{kb})$. [1] The genome of these viruses includes a 5 'cap structure and a 3' poly (A) tail, which gives them the ability to act as mRNA to translate replication polyproteins. The replication gene, which encodes non-structural proteins, as opposed to structural and accessory proteins, which make up only about 10 kilobases $(\mathrm{kb})$ of the viral genome, is two-thirds of the genome, about 20 kilobases (kb). [3] The coronavirus has club-like spikes from its surface. [4] The proteins that contribute to the overall structure of all coronaviruses are the spike (S), envelope (E), membrane (M), and nucleocapsid $(\mathrm{N})$, all of which are encoded at the 3 'end of the viral genome. In the specific case of the SARS coronavirus, a defined receptor-binding domain of the $\mathrm{S}$ protein mediates the attachment of the virus to its cellular receptor, angiotensinconverting enzyme-2 (ACE-2). Some coronaviruses, especially the members of subgroup $\mathrm{A}$, and beta coronaviruses also have a shorter spike-like protein called hemagglutinin esterase (HE) [5]. Coronaviruses can be divided into 4 genera: alpha, beta, delta, and gamma, of which alpha and beta $\mathrm{CoV}$ are known to infect humans. [3] 


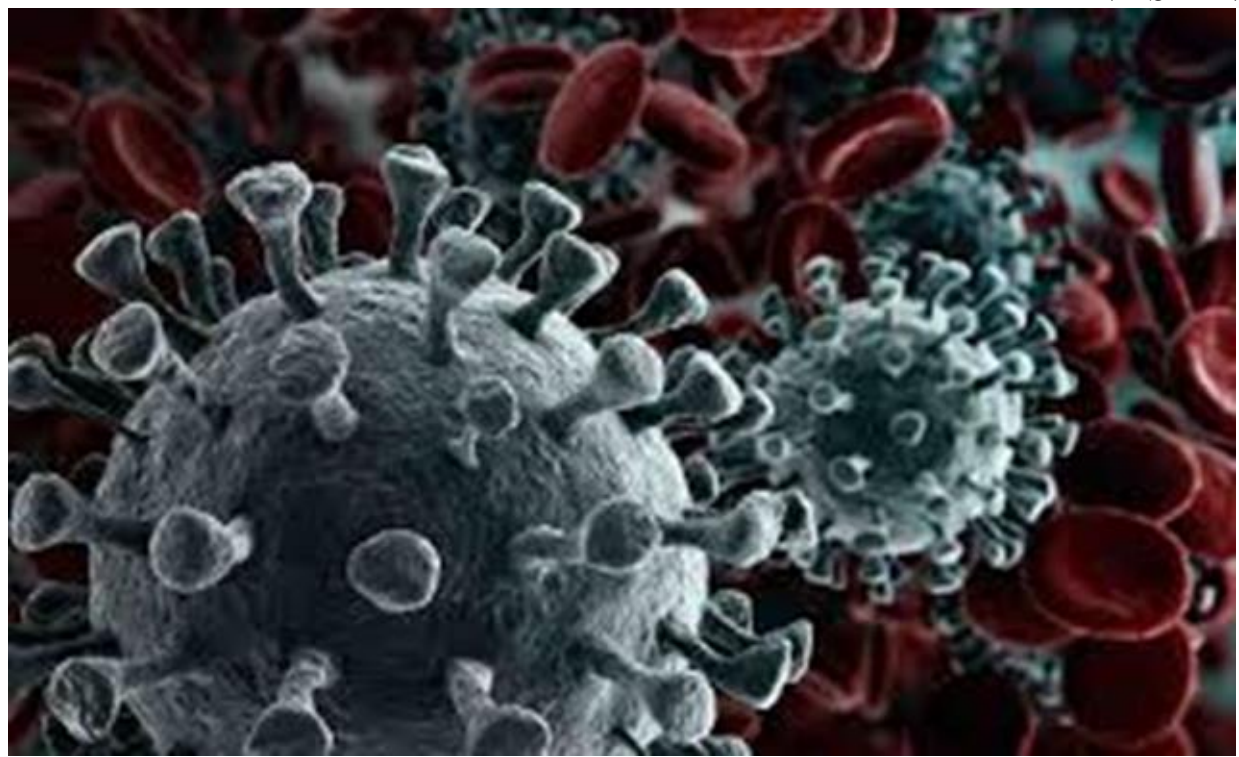

Figure 1. Electron microscope picture of COVID-19.

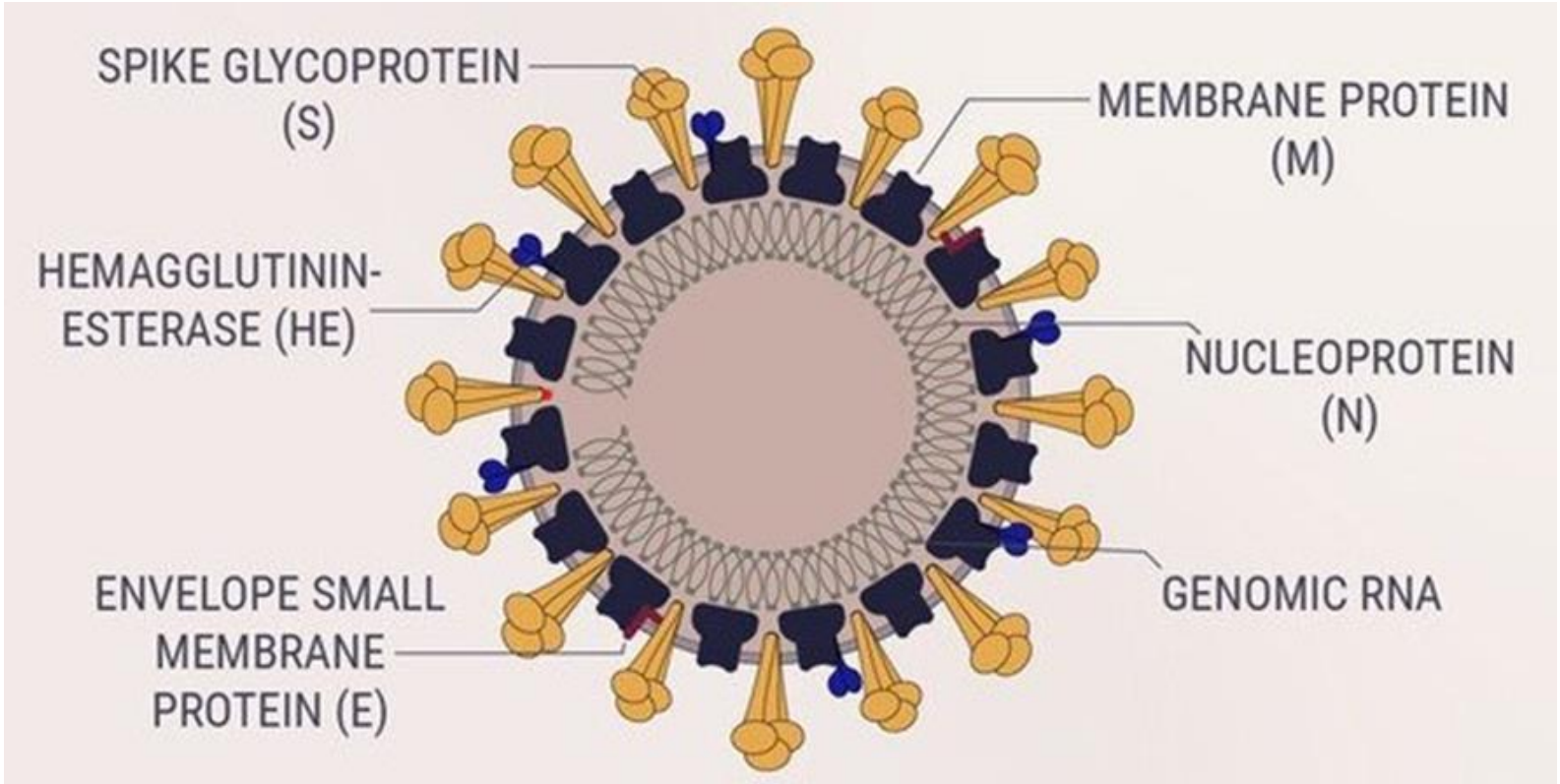

Figure 2. Structure of COVID

So far seven types of coronavirus have been found in humans: HCoV-229E, HCoV-OC43, HCoV-NL63, HCoV-HKU1, SARS-CoV, MERS-CoV, CoV-ALFA-SARS или 2019 nCoV. [6]

SARS-CoV-2 belongs to a coronavirus strain associated with the severe acute respiratory syndrome (SARSr-CoV) and is a genus of beta coronavirus. [7] Recent studies have shown that SARS-CoV-2, known as a novel virus, can share $79.5 \%$ of its genetic sequence with SARS-CoV and $96.2 \%$ is homologous to bat coronavirus genome named RaTG13, but the synonymous mutation also results in increased $\mathrm{T}: \mathrm{C}$ transition. This mutation may be due to the loss of RNA 3'-to-5' exoribonuclease function. The 2019-nCoV shares an ACE2-like entry cell receptor with SARS-CoV-2 which indicating that SARS-CoV-2 may be more infectious to humans than SARS-CoV. [8, 9]

\section{EPIDEMIOLOGY}

Epithelial cells are the first cells in the human body to be infected with the coronavirus. In them, the coronaviruses are located between the endoplasmic reticulum and the Golgi apparatus. After replication of viral particles with reverse genetic systems, the viruses are transmitted to the plasma membrane by a secretory pathway and released by exocytosis. [10] 
Of the four known major coronavirus proteins, the $\mathrm{S}$ glycoprotein mediates binding to the host epithelial cell receptor. The $M$ protein enhances the curvature of the membrane and attaches to the nucleocapsid, determining the shape of the virus. E protein facilitates the assembly and release of the virus, but it also has other functions such as ion channel activity. The $\mathrm{N}$ protein helps to bind the viral genome to the replicase-transcription complex and wraps the encapsulated genome into the viral particles. Haemagglutinin esterase binds sialic acid to surface glycoproteins through its acetyltransferase activity. [1, 11]

The incidence of ill children under the age of 18 in the Wuhan epidemic was $2.4 \%$ of the total number reported. [12] Observations showed that the incubation period was 1-14 days, average 5-6 days, although in some cases the incubation took 24 days, which is more than the WHO statistics. The number of reproductions (RO) for SARS-CoV-2 is between 2 and 3, which indicates that the epidemic potential of this disease is higher than SARS with RO 0.8 and MERS-RO 0.69 $[13,14]$. The mortality rate for CoVID is between 2.3-3\%. [15, 16] The coronavirus reservoir is different. In SARS, cats are the source of infection, however, for CoVID it is still not clear. Moreover, pangolins and snakes are thought to host SARS-CoV19 mediators. $[17,18]$

Coronaviruses are transmitted from person to person through sneezing and coughing, through airborne droplets from the nasal mucosa. $[19,20]$ There is no convincing evidence that the CoVID-19 virus is transmitted in the air, but perhaps respiratory droplets, containing the virus, remain on the surfaces and contribute to the transmission of the virus. [21]

\section{CLINICAL MANIFESTATION}

Depending on the severity of the clinical manifestation, symptoms can occur in four levels: mild, moderate, severe, and critical. In those with a severe clinic, one of the three criteria is seen: respiratory rate above $30 / \mathrm{min}$, oxygen saturation below 93\%, $\mathrm{PaO} 2 / \mathrm{FiO} 2$ $<300 \mathrm{mmHg}$. In most cases, children with CoVID infection are asymptomatic or have mild symptoms without fever and pneumonia. $[22,23]$ In some cases patients have a fever, fatigue, cough, nasal congestion, runny nose,
VASILEVA A., et al. expectoration, diarrhea, headache. Dyspnea and cyanosis are symptoms accompanying systemic toxic manifestations such as weakness or anxiety, malnutrition, poor appetite, decreased activity, and, after one week, manifestations of respiratory failure. In the most severe cases, septic shock, metabolic acidosis, irreversible bleeding, and coagulation dysfunction occur. In critically ill patients, severe respiratory failure, septic shock, and multiorgan failure occur. [24] Significantly better prognosis and improvement, as well as shorter disease duration, are explained by the reduced ACE-2 expression in the lungs. In children, these receptors are mainly located in the epithelial cells in the upper airways. With age, ACE-2 receptors play an important role in lung defense mechanisms. [25]

\section{LABORATORY TESTS}

Laboratory tests show leukopenia, lymphopenia, decreased hepatic and myoglobin enzymes, thrombocytopenia. [26] LDH and CRP levels increase and decrease more than in adult patients, especially in the most severe cases. [23, 26]

Elevated CRP and ESR values have also been observed in the second phase of the disease, as well as severe lymphopenia. In most cases, procalcitonin was normal. [27, 28] Genetic sequencing is the main method of proving the infection. RT-PCR, based on the $\mathrm{S}$ and $\mathrm{N}$ genes, is used to detect viral RNA and is defined as the gold standard. [7, 24, 29, 30] Xrays of the lungs are also needed to diagnose the disease. The most common changes are bilateral ground-glass opacities, with or without consolidation in the pulmonary periphery. In severe lung infections, numerous lobar lesions are visualized in both lungs. [27] In a CT scan of the lungs, lung changes are similar to those in adults, but to a relatively mild degree. [31]

\section{DIAGNOSIS}

- Epidemiologic criteria

- Laboratory results

- X-ray or CT of the lungs

However, infants should have the following symptoms: unstable body temperature, low activity with proper nutrition, shortness of breath, bilateral changes such as ground-glass opacities on the radiography. 
VASILEVA A., et al.

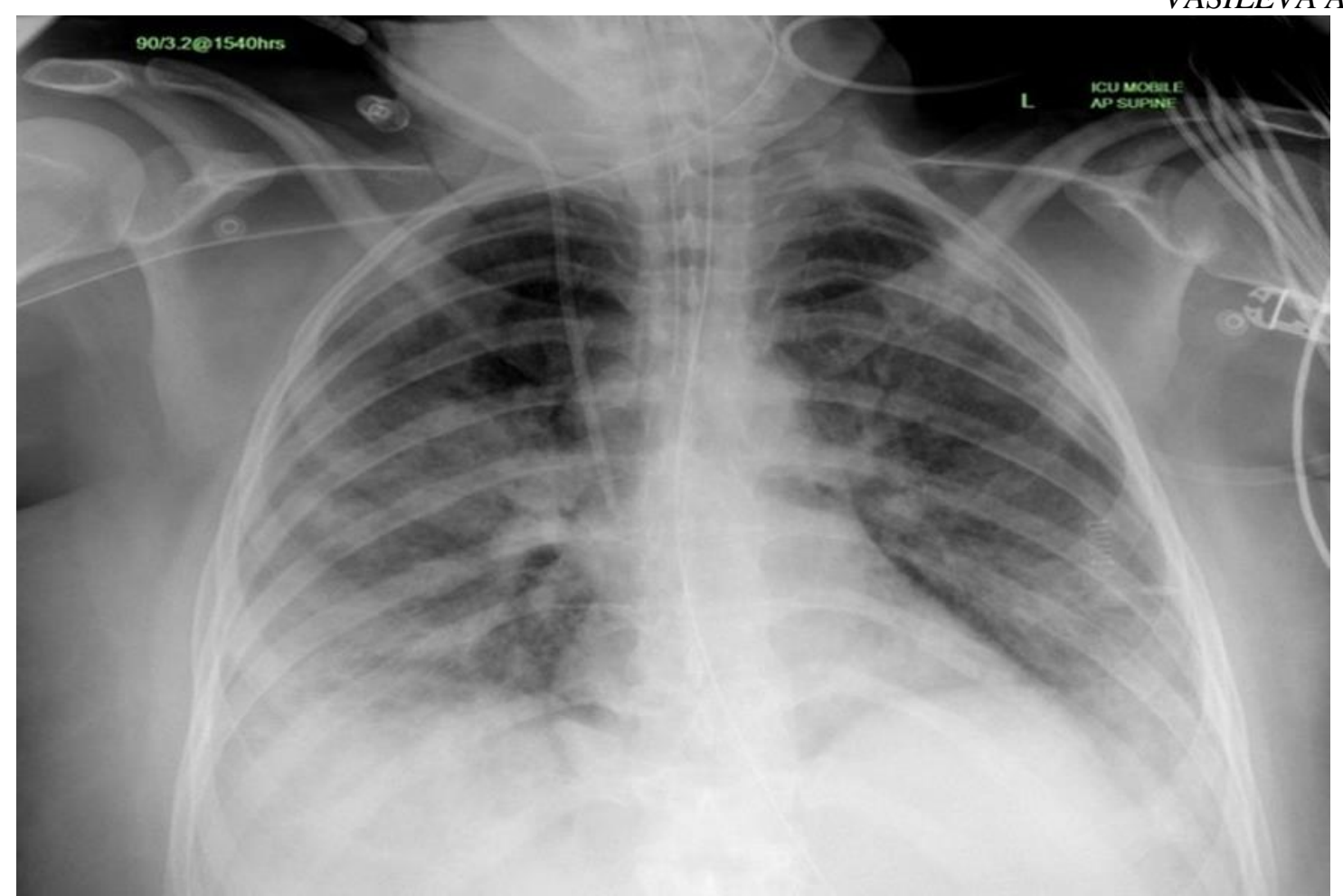

Figure 3. Chest radiography of a patient with COVID-19. [32]

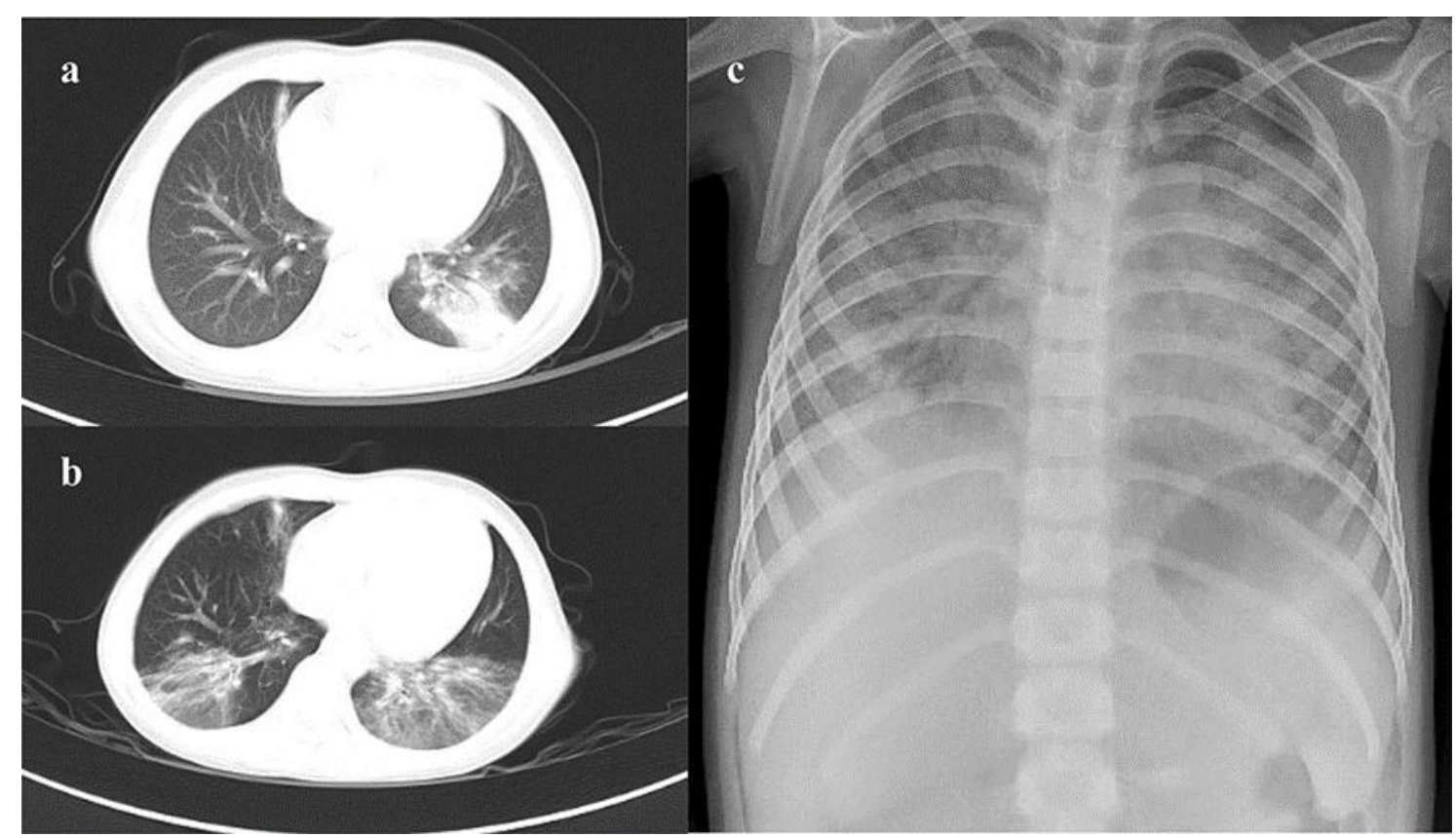

Figure 4. CT and chest radiography of patient, diagnosed with COVID-19, showing consolidation and groundglass opacities. [33]

\section{TREATMENT}

No specific treatment has been identified yet. Bed rest and supportive care are needed. If necessary - correction of water-electrolyte homeostasis. In a study of the serine protease TMPRSS2, which is required for protein $\mathrm{S}$ priming, it was suggested that the homostat mesylate, as a protease serase inhibitor, which could prevent COVID 19 from entering the lung cells. [34]

\section{Antiviral therapy}

According to studies of ZM Chen et al. [28], as well as the study by K Shen et al. [27] some effective antiviral agents that can be used are interferon $a 2 b \quad$ (nebulization), lopinavir (ritonavir). The use of ganciclovir and ribavirin is not recommended as their effect has not been established. In some studies, double-stranded activated caspase, an oligomerizer (DRACO), and immucillin A, which inhibit RNA, were used as antiviral drugs. [3] In a study, including 
VASILEVA A., et al.

nine children, of Rahimzadeh $G$ et al. [12] the use of oseltamivir, lopinavir, and ritonavir gave excellent results, with the benefits of oseltamivir being most pronounced. In the most severe cases, intravenous immunoglobulin might be used. [11, 27, 12, 23, 35]

\section{Antibacterial therapy}

The use of meropenem, vancomycin, chloroquine has been proven to have a good effect. [12] In a study by Q Lu et al. [36] the combination of sirolimus and dactinomycin was used. [12]

\section{Other medications}

Q Lu offers two combinations of drugs: mercaptopurine plus melatonin and torimiphene plus imudine. [36]

\section{Oxygen therapy}

An indication of oxygen therapy is hypoxia. Its application, as well as mechanical ventilation, has led to an improvement in the condition of sick children. [27, 12, 23, 28] In another study of Q Lu, in children with ARDS, surfactant, inhaled NO, high-frequency oscillatory ventilation, and pulmonary extracorporeal membrane were used successfully. [36] In 6 studies, the serums from CoVID-19 patients were found to be useful for treatment. [36]

\section{Corticosteroids}

Regarding the use of corticosteroids - they could be used in limited cases in ARDS, septic shock, encephalopathy, and hemophagocytic syndrome. The recommended drug is intravenously administered methylprednisolone $1-2 \mathrm{mg} / \mathrm{kg}$ for $3-5$ days. [27, 28]

K Shen used oral ibuprofen at a dose of 5-10 $\mathrm{mg} / \mathrm{kg}$, as well as acetaminophen orally at a dose of $10-15 \mathrm{mg} / \mathrm{kg}$. [27]

\section{CONCLUSIONS}

Although little is already known about CoVID19 infection, it still raises many questions that cannot be answered accurately. It is an indisputable fact that it is highly contagious. Despite the relatively small number of sick children, they are also at risk. The risk of severe complications of the pulmonary system is a challenge. Due to the lack of causal treatment, the possibilities for the use of a small number of antiviral drugs and those for maintenance treatment require precise diagnosis, accurate assessment of clinical development, and expected complications. Thus, increasing knowledge, based on world experience, will reduce the risk of omissions, as well as shorten the recovery period for those infected.

\section{REFERENCES}

1. Fehr, A.R. and S. Perlman, Coronaviruses: an overview of their replication and pathogenesis, in Coronaviruses. 2015, Springer. p. 1-23.

2. Chen, H., et al., Clinical characteristics and intrauterine vertical transmission potential of COVID-19 infection in nine pregnant women: a retrospective review of medical records. The Lancet, 2020.

3. Zhao, L., et al., Antagonism of the interferon-induced OAS-RNase L pathway by murine coronavirus ns 2 protein is required for virus replication and liver pathology. Cell host \& microbe, 11(6): p. 607-6162012.

4. Phan, T., Novel coronavirus: From discovery to clinical diagnostics. Infection, Genetics and Evolution, 2020. 79: p. 104211.

5. Hiroi, S., T. Kawahata, and K. Furubayashi, First isolation of human adenovirus type 85 by molecular analysis of adenoviruses in cases of urethritis. Journal of Medical Microbiology, 2020: p. jmm001149.

6. King, A.M., et al., Virus taxonomy: ninth report of the International Committee on Taxonomy of Viruses. Vol. 9. 2011: Elsevier.

7. Zhou, P., et al., A pneumonia outbreak associated with a new coronavirus of probable bat origin. Nature, 2020: p. 1-4

8. del Rio, C. and P.N. Malani, 2019 Novel coronavirus - important information for clinicians. JAMA, 2020.

9. Lv, L., et al., Comparative genomic analysis revealed specific mutation pattern between human coronavirus SARS-CoV-2 and Bat-SARSr-CoV RaTG13. BioRxiv, 2020.

10.Pratelli, A., Basic science track entry and release of canine coronavirus from polarized epithelial cells. Microbiologica-Quarterly Journal of Microbiological Sciences, 2011. 34(1): p. 25.

11. Dhama, K., et al., Coronavirus Disease 2019-COVID-19. 2020. 
12. Rahimzadeh, G., et al., COVID-19 Infection in Iranian Children: A Case Series of 9 Patients. JPR, 2020. 8(2): p. 139-144

13. del Rio, C. and P.N. Malani, COVID-19New Insights on a Rapidly Changing Epidemic. JAMA, 2020.

14. Breban, R., J. Riou, and A. Fontanet, Interhuman transmissibility of Middle East respiratory syndrome coronavirus: estimation of pandemic risk. The Lancet, 2013. 382(9893): p. 694-699.

15. Zhang, C., L. Shi, and F.-S. Wang, Liver injury in COVID-19: management and challenges. The Lancet Gastroenterology \& Hepatology, 2020.

16. Novel, C.P.E.R.E., The epidemiological characteristics of an outbreak of 2019 novel coronavirus diseases (COVID-19) in China. Zhonghua liu xing bing xue za zhi= Zhonghua liuxingbingxue zazhi, 2020. 41(2): p. 145.

17. Lam, T.T.-Y., et al., Identification of 2019nCoV related coronaviruses in Malayan pangolins in southern China. bioRxiv, 2020

18. Ji, W., et al., Homologous recombination within the spike glycoprotein of the newly identified coronavirus may boost crossspecies transmission from snake to human. J. med. virol, 2020.

19. Sarkar, B., et al., The Essential Facts of Wuhan Novel Coronavirus Outbreak in China and Epitope-based Vaccine Designing against 2019-nCoV. BioRxiv, 2020.

20. Drosten, C., et al., Transmission of MERScoronavirus in household contacts. New England Journal of Medicine, 2014. 371(9): p. 828-835.

21. Ong, S.W.X., et al., Air, Surface Environmental, and Personal Protective Equipment Contamination by Severe Acute Respiratory Syndrome Coronavirus 2 (SARS-CoV-2) From a Symptomatic Patient. JAMA, 2020.

22. Shen, K.-L. and Y.-H. Yang, Diagnosis and treatment of 2019 novel coronavirus infection in children: a pressing issue. 2020, Springer.

23. Du, W., et al., Clinical Characteristics of COVID-19 in Children Compared with Adults Outside of Hubei Province in China. Available at SSRN 3546097, 2020.
VASILEVA A., et al.

24. Wang, Y., et al., Unique epidemiological and clinical features of the emerging 2019 novel coronavirus pneumonia (COVID-19) implicate special control measures. Journal of Medical Virology.

25. Lee, P.-I., et al., Are children less susceptible to COVID-19? Journal of Microbiology, Immunology and Infection, 2020.

26. Zimmermann, P. and N. Curtis, Coronavirus Infections in Children Including COVID-19. The Pediatric Infectious Disease Journal, 2020. 1.

27. Shen, K., et al., Diagnosis, treatment, and prevention of 2019 novel coronavirus infection in children: experts' consensus statement. World Journal of Pediatrics, 2020: p. 1-9.

28.Chen, Z.-M., et al., Diagnosis and treatment recommendations for pediatric respiratory infection caused by the 2019 novel coronavirus. World Journal of Pediatrics, 2020: p. 1-7.

29. Lu, R., et al., Genomic characterisation and epidemiology of 2019 novel coronavirus: implications for virus origins and receptor binding. The Lancet, 2020. 395(10224): p. 565-574.

30.Chu, D.K., et al., Molecular diagnosis of a novel coronavirus (2019-nCoV) causing an outbreak of pneumonia. Clinical chemistry, 2020.

31. Li, W., et al., Chest computed tomography in children with COVID-19 respiratory infection. Pediatric Radiology, 2020: p. 14.

32. https://radiopaedia.org/cases/covid-19pneumonia-paediatric?lang=us

33. https://link.springer.com/article/10.1007/s1 2519-020-00354-4

34.Hoffmann, M., et al., SARS-CoV-2 Cell Entry Depends on ACE2 and TMPRSS2 and Is Blocked by a Clinically Proven Protease Inhibitor. Cell, 2020.

35. Tang, A., et al., A retrospective study of the clinical characteristics of COVID-19 infection in 26 children. medRxiv, 2020

36.Lu, Q. and Y. Shi, Coronavirus disease (COVID-19) and neonate: What neonatologist need to know. Journal of Medical Virology, 2020. 